\title{
Mechanism of Colistin Resistance to Acinetobacter Baumannii and its Progress: A Review Article
}

\author{
Zhang Bailing, Zhang Jieling* and Li Honglang* \\ The Second Affiliated Hospital of Nanchang University, Department of Laboratory Medicine, China
}

*Corresponding author: Zhang Jieling, Li Honglang, The Second Affiliated Hospital of Nanchang University, Department of Oncology, China

\begin{abstract}
ARTICLE INFO
Received: 㓞 July 16, 2020

Published: 幽 July 27, 2020

Citation: Zhang Bailing, Zhang Jieling, Li Honglang. Mechanism of Colistin Resistance to Acinetobacter Baumannii and its Progress: A Review Article. Biomed J Sci \& Tech Res 29(1)-2020. BJSTR. MS.ID.004755.

Abbreviations: XDR: Extensively Drug-Resistant, MDR: Multidrug-Resistant, OM: Outer Membrane, DAB: Diaminobutyric Acid, PDR: Pandrug-Resistance, PP: Phosphate Pathway, TCA: Tricarboxylic Acid

\section{ABSTRACT}

Deemed as an important opportunistic pathogen bacterium, Acinetobacter baumannii can be widely found in nature and shows a strong capability of acquired resistance and transmissibility. Most of its strains can be grouped as either MultidrugResistant (MDR) or Extensively Drug-Resistant (XDR) in nature and lead to lower respiratory tract infection, bloodstream infection, and urinary system infection, as well as intraabdominal infection, which are less treatable. Colistin resistance is primarily a result of phosphoethanolamine addition into lipid A via phosphoethanolamine transferase. The enzyme is chromosomally encoded by the pmrC gene, which is, in turn, modulated by a two-component system PmrA/PmrB. In the context of A. baumannii, its clinical isolates reveal the key mechanism of resistance against colistin involving mutations in the $p m r A, p m r B$, or $p m r C$ genes, thereby resulting in $p m r C$ overexpression. This review aimed to contribute towards a better comprehension of the pathogen by summarising A. baumannii and its resistance mechanism against colistin. It also emphasised the consequences of colistin resistance-correlated mutations and the biological pathogenic response that emerged.
\end{abstract}

Keywords: Colistin; Acinetobacter baumannii; Antibiotic Resistance; Multidrug Resistance

\section{Introduction}

Acinetobacter baumannii is ubiquitous in nature and classified as non-fermented gram-negative bacilli. As an important conditional pathogen of nosocomial infections, it mainly causes reduced breath rate, tract infection, bloodstream infection, urinary system infection, and abdominal cavity infection, among others. Most of the conducted studies such as new epidemiological surveys have suggested its emergence as the pathogenic bacteria of hospitalacquired pneumonia. Besides, it is frequently linked to pneumonia and septicaemia cases among those with impaired immunity [1]. Similarly, the rate of antibiotic resistance in the past few years has increased significantly, which can be especially seen in secondgeneration cephalosporins, third-generation cephalosporins, carbapenems, aminoglycosides, and quinine. Moreover, most of the known A. baumannii strains are either multidrug-resistant (MDR) or extensively drug-resistant (XDR) in nature [1]. Currently, almost no medicine available is available for them. In general, the microorganisms may undergo different mechanisms to induce their adaptation against varying antimicrobial agents and environmental stimulants. Meanwhile, bacteria are subjected to genetic modifications in order to generate genes that are capable of overcoming antibiotic actions due to enhanced performance. In particular, such modifications occur in a negligible amount of base pairs in the DNA, thereby resulting in one or multiple amino acids replacement in an essential target.

Possibly seen in locations such as cell structure or cell wall and enzymes, the modifications can yield newly-emerged resistant strains [2]. A baumannii shows a robust growth on regularly employed laboratory media, such as blood agar, chocolate agar, and MacConkey agar. Its growth on blood agar yields colourless, nonhaemolytic, and shiny mucoid colonies, which show a smoothened 
texture and a diameter of $1-2 \mathrm{~mm}$ following incubation for 1824 hours at the temperature of $37^{\circ} \mathrm{C}$. Meanwhile, its colonies MacConkey agar are tomb-shaped despite being similarly shiny and mucoid in nature, which delineates its non-lactose fermenting capability. In contrast, growth on the selective agar of Leeds Acinetobacter Medium yields pink-coloured colonies if cultured with a supplement present [3]. In general, Acinetobacter spp. are known as free-living saprophytic organisms, which are extensively found across varying environments, such as the soil, water, wastewater, vegetables, and animal and human skin [4].

Their isolation can be done from different body parts of a healthy individual, ranging from the nose, ear, throat, forehead, trachea, and conjunctiva to the vagina and perineum, axillae, groin, hands, and toe webs. However, a majority of these strains are not A. baumannii, but rather other strains [5]. In a hospital setting, the organisms can be isolated from the beds, curtains, walls, roofs, medical devices, and equipment. Moreover, they can be found on other communal surfaces, such as healthcare personnel belongings, tap water sinks, telephones, door handles, hand sanitisers, dispensers, trolleys, bins, and computers. Additionally, their survival on inanimate objects may last a prolonged duration. Therefore, their prevalence in such setting is driven by attributes such as their resistance against essential antimicrobial drugs and disinfectants, and their capacity of survival in desiccants [6]. Furthermore, the emergence of XDR strains has resulted in the human clinical usage of colistin, which is an "old" antibiotic dating back to the 1960s.

The antibiotic utilisation in human therapy was stopped as a result of its nephrotoxicity and neurotoxicity attributes, especially during a period of time in which the emerging new alternatives yielded good antibacterial potency and fewer toxicity outcomes. Following this, colistin is primarily implemented in the form of topical formulations. However, the materialisation of XDR strains has driven physicians to implement the antibiotic as one of the alternative curative alternatives against applicable cases of infection. After its re-introduction in human clinical practice, colistin-resistant strains have emerged and are detailed accordingly [7]. At present, the mechanisms of resistance employed by A baumannii are linked with chromosomal encoding in nature [8].

\section{What We Know about Colistin Mechanism of Action}

Colistin can be described as a bactericidal polycationic lipopeptide, which is also referred to as polymyxin E. It consists of a cyclic decapeptide tethered to a fatty acid chain and is known as a narrow-spectrum antimicrobial agent yielding notable activity against a majority of the Enterobacteriaceae family members. They include Escherichia coli, Enterobacter spp., Klebsiella spp., Citrobacter spp., Salmonella spp., and Shigella spp. Furthermore, colistin offers antibacterial action against certain typical non-fermentative Gramnegative bacteria, some examples being A. baumannii, Pseudomonas aeruginosa, and Stenotrophomonas maltophilia [9]. Meanwhile, the antibiotic is associated with the presence of hydrophilic and lipophilic moieties both, which are cationic regions capable of interacting with the bacterial outer membrane. The interaction occurs via the displacement of magnesium and calcium bacterial counter ions present in the lipopolysaccharide. Moreover, the hydrophobic/hydrophilic regions generate interactions with the cytoplasmic membrane as well, yielding a detergent-like effect by solubilising the membrane within an aqueous setting. Therefore, its impacts are bactericidal in nature even if they occur in an isosmolar environment [10].

Initially, colistin aims for the cellular target, namely the polyanionic LPS, which is a constituent of the Gram-negative bacterial Outer Membrane (OM). The antibiotic is equipped with an amphiphilic attribute, which is essential for it to interact with the hydrophobic lipid A (i.e. a constituent of LPS). Lipid A, in particular, plays a key role in controlling the cell permeability [11]. Furthermore, molecule destabilisation occurs following divalent cation $\mathrm{Ca}_{2}+$ and $\mathrm{Mg}_{2}+$ displacement, which is caused by electrostatic interaction between positively-charged $\alpha, \gamma$-Diaminobutyric Acid (Dab) residues of colistin and negatively-charged phosphate groups of lipid A. The three-dimensional structure then undergoes a modification, thereby triggering certain areas of the $\mathrm{OM}$ and their permeability. This leads to a facilitated passage for colistin via a mechanism of self-promoted uptake [12]. Following this, the antibiotic will destruct the bacterial membrane, which causes the cytoplasmic content to leak and ultimately, result in cell death [11].

\section{Discussion}

Mechanism of resistance for colistin against A. baumannii: Several mechanisms of acquired resistance for colistin are detailed accordingly in this work. The five mechanisms are as follows: 1) engaging the mutations that occur in lipid $A$ encoding genes (i.e. IpxA, lpxC, and lpxD); 2) total LPS loss; 3) pmrA and pmrB point mutations; 4) efflux pump transporter upregulation; and 5) use of plasmid-mediated colistin resistance gene. Accordingly, the first mechanism occurs via the addition of phosphoethanolamine (pEtN) due to $\mathrm{pmrA} / \mathrm{pmrB}$ two-component system mutations. This results in LPS loss, the outer component of Gram-negative organisms, and the preliminary colistin target [13]. Next, the second mechanism occurs as a result of defective lipid A synthesis, thereby yielding a complete LPS loss [13], whereas the third mechanism describes the manner in which pmrA and pmrB point mutations cause their gene expression to be up-regulated. This mechanism remodels the outer membrane accordingly. Meanwhile, the fifth mechanism is recently described following a scholarly discovery of plasmidmediated colistin resistance gene, mcr-1, in E. coli [14]. Despite the gene is not identified in A. baumannii, speculations have arisen that the shift from MDR Acinetobacter to Pandrug-Resistance (PDR) cannot be avoided as a result of transmissible colistin resistance mechanisms that emerge [15]. 


\section{LPS Modification Mediated by Two-Component System pmrA/pmrB}

As the commonest resistance mechanism against colistin, LPS modification via phosphate group substitution with molecules conferring a positive charge to the LPS hinders colistin binding from occurring. The pmrA and/or pmrB mutations (typically seen in pmrB more) in the case of $A$. baumannii can trigger pmrA's constitutive expression, resulting in an up regulated pmrCAB operon. Consequently, pEtN is synthesised and added to the $4^{\prime}$-phosphate or $1^{\prime}$-phosphate of lipid A [16]. In contrast, a resistant phenotype may become a susceptible version following the compensatory mutations occurring in the pmr locus, thus either minimising the pmrCAB operon hyper-activation or causing the pmr gene to transform into a non-mutated structure [17]. Regardless, certain strains are capable of sustaining the pmrB mutation without requiring auxiliary compensatory mutations. Furthermore, from 30 colistin-resistant $A$. baumannii strains, six have been found to show no evidence of mutation in their respective pmrA/pmrB loci. Therefore, this is suggestive of other gene involvement towards achieving colistin resistance, resulting in the PmrAB two-component system to over-express itself. Additionally, overexpression of both genes is seemingly crucial for the purpose of colistin resistance, which contrasts the amino acid changes [18].

Besides, alternative lipid A structure modifications have been identified in clinical and laboratory colistin-resistant strains with a diphosphoryl hepta-acylated lipid A structure, namely pEtN and galactosamine (GalN) modifications both [19]. The hepta-acylation of lipid A offers defensive advantages against cationic antimicrobial polypeptides, which include polymyxins. In E. coli and Salmonella spp., reinforcement of the LPS portion in the OM barrier is possible due to enhanced OM acyltransferase PagP production, thereby yielding the preservative hepta-acylated lipid A attribute. In contrast, A. baumannii lacks the pagP gene and thus establishes its own PagPindependent mechanism so as to synthesise its preservative heptaacylated lipid A. Accordingly, the characterisation of two putative acyltransferases (i.e. designated as LpxLAb and LpxMAb) occurs in which one and two lauroyl (C12:0) acyl chains are respectively transferred in the process of biosynthesising lipid A. In particular, the LpxMAb-dependent acylation of lipid A has been found to be crucial in ensuring the A. baumannii strain survival in desiccation conditions [20].

\section{Loss of LPS}

In general, LPS synthesis occurs in the cytoplasm via the Lpx pathway, whereby its translocation to the OM proceeds using the Lpt pathway. Therefore, its complete loss has been noted in A. baumannii strains that are resistant to colistin. This emerges either due to mutations by nucleotide substitution, deletion, or insertional inactivation processes via the insertion sequence ISAba11, which is seen in the IpxA, IpxC, and lpxD genes [21]. Furthermore, colistin resistance is further correlated with the supplementary lpsB gene mutations, glycosyltransferase encoding, and its involvement in the LPS core synthesis. Besides, a high resistance is further noted when lipid A is absent, where it functions as the antibiotic's preliminary target [22]. Similarly, its absence in the $\mathrm{OM}$ as a result of genetic mutations for the genes tasked with encoding the OM protein LptD; it is responsible the LPS molecule's last translocation following its synthesis [23].

The bacteria lacking LPS is capable of modifying the host innate immune inflammatory response activation [24]. Recently, a study has differentiated the metabolome of polymyxin-susceptible and polymyxin-resistant $A$. baumannii strains, yielding three drastically varied metabolic profiles. They are: (a) specific amino acid and carbohydrate metabolite modification, specifically those of the pentose Phosphate Pathway (PPP) and Tricarboxylic Acid (TCA) cycle intermediates; (b) lower nucleotide levels in the LPS-deficient strains; and (c) higher prevalence of short-chain lipids as opposed to the parent polymyxin-susceptible ATCC 19606 (American Type Culture Collection) [25].

\section{Gene Involvement in Outer Membrane Asymmetry}

The shifting antimicrobial susceptibility against colistin may be attributed to mutations in other genes, instead of pmrAB and IpxACD. In general, the OM of Gram-negative bacteria is associated with an asymmetric lipid distribution, which is crucial towards its role in ensuring cell integrity and being a barrier. Therefore, LPS distribution can be disrupted due to phospholipid build-up in its outer leaflet, further increasing the cellular lability against small and toxic molecules. In particular, the OM lipoprotein VacJ is a component of the Vps-VacJ ABC transporter system, which is tasked with sustaining the phospholipid concentration in the $\mathrm{OM}$ inner leaflet and the LPS in the OM outer leaflet (i.e. OM asymmetry) [26]. Moreover, the PldA phospholipase action is further enhanced in bacteria cells having destabilised membrane; this seemingly causes the removal of phospholipids from the OM outer leaflet inwards to ensure the asymmetry [27]. Additionally, it has been recently suggested that the vacJ and pldA genes both may potentially bring about the colistin resistance in A. baumannii. These genes are the location where the mutation occurs and their role can be linked to maintaining an asymmetrical OM [28].

\section{Efflux Pumps}

Some works have indicated the potential involvement of efflux pumps towards colistin resistance in A. baumannii. In particular, the upregulation of 18 genes encoding putative efflux transporters has been found following the presence of physiological $\mathrm{NaCl}$ concentration, which further allows tolerance for various antibiotics, colistin included [29]. Furthermore, another recent work has linked the application of efflux inhibitors such as cyanide 3-chlorophenylhydrazone (CCCP) towards a remarkably significant reduction of colistin's minimal inhibitor concentrations. Therefore, 
this is strongly suggestive of the efflux pump's role in its resistance phenotype [30].

\section{Plasmid-Mediated Colistin Resistance}

Plasmid-mediated colistin has emerged as a notable obstacle and worldwide issue as the colistin-resistant genes are easily transferred to any susceptible strains [14]. In general, the mcr genes are correlated with the colistin resistance and its horizontal transfer. They were first described in November 2015 through E. coli isolates obtained from pigs and meat in China [14]. The mcr gene is under the umbrella of the pEtN enzyme family, whereby its expression causes pEtN to be added into lipid A. Literature has shown that the isolates with the mor1 gene reveal colistin resistance without any other resistance mechanisms as they yield 4-8 times increment of colistin minimum inhibitory concentration (MIC) [31]. Additionally, it should be noted that mcr1 gene production causes lysozymic resistance as well [32]. Following its preliminary findings, different regions have recorded the presence of mcr1mediating transferable colistin resistance, such as Europe, Asia, the Americas, and Africa [33,34]

Scholars have further hypothesised that the mor1 gene is originally found in animals, especially pigs and cattle, which then disseminates to humans despite a lower percentage of mcr1-positive isolates in humans in comparison with animals [35]. Regardless, the transmissible gene has been recorded to encompass the wide-ranging genera of Enterobacteriaceae, thereby inclusive of E. coli, Klebsiella spp., Enterobacter spp., Salmonella spp., Shigella spp., and Cronobacter spp. However, most of its presence is noted in E. coli. Furthermore, certain mcr1-containing plasmids can transfer other genes showing resistance against other antibiotics, which include $\beta$-lactams, aminoglycosides, quinolones, sulfonamides, tetracyclines, and fosfomycin [36]. Additionally, the mcr gene is notably found in Enterobacteriaceae isolates and carries carbapenemase genes, such as blaNDM1, blaNDM5, blaNDM9, blaOXA48, blaKPC2, and blaVIM1 $[37,38]$.

Besides, a new report by Xavier et al. has delineated the discovery of ground-breaking plasmid-mediated colistin resistance gene otherwise identified as mcr2 in E. coli [39]. Following this, the discovery of mcr3 and mcr4 genes has been reported [39,40] finally detailed a novel gene from the mcr family in Salmonella paratyphi B in July 2017, which is transported in transposons rather than plasmids. Afterwards, 2018 marked the discovery of three mobile colistin-resistance genes, namely mcr6, mcr7, and mcr8. Meanwhile, AbuOun et al. have identified a novel form of mor2 from Moraxella pluranimalium, which is renamed as mcr6.1, and indicated the potential role of Moraxella spp. as an innate repository of mor and mcr-harbouring Moraxella in pig populations [41]. Next, Yang et al. have distinguished $K$. pneumoniae isolates harbouring a novel $\mathrm{mcr}$ variant then renamed as mcr7.1, which are obtained from chickens in China [42]. Similar to mcr3, the scholars have suggested that mcr7 originates from Aeromonas spp [42]. due to its structure and $78 \%$ of nucleotide identity similarities with the mcr3 gene.

Last but not least, mcr8 is the newest mobile genetic element identified, which is discovered in K. pneumonia isolates. However, its identification occurs with mcr8 and carbapenemase-encoding gene blaNDM concurrently, which has thus emerged as an issue [43]. Mcr8 has been notably present for some duration of time and distributed in K. pneumonia [43]. Meanwhile, mcr2-8 genes show similarities with mcr1 in which the presence of pEtN results in leads to phosphoethanolamine addition into lipid A and consequently, causes colistin resistance. In contrast, mcr 1 and mor 2 genes both have come from the Moraxella spp., whereas the mcr3 and mcr4 genes closely parallel pEtN from Aeromonas spp. and Shewanella frigidimarina, respectively [44], while mor5 gene's origin is still unknown [40]. Additionally, despite mcr being a plasmid-mediated gene, Zurfluh et al. have recently discovered mor1 gene on the chromosomes of E. coli strains. Therefore, this births the hypothesis suggesting the gene's integration into the genome of certain isolates [45].

\section{Conclusion}

In conclusion, $A$. baumannii has been ultimately underlined as a nosocomial pathogen capable of yielding a high level of resistance against different antibiotics. Furthermore, XDR and PDR pandrug-resistant isolates have been consistently detailed in multiple medical facilities. Following this, the historicallyabandoned antibiotic colistin has emerged as the final option of suggested antimicrobial, which is followed by multiple works revealing its resistance as highly prevalent and rapidly increasing in A. baumannii. Therefore, clinicians must remain alert regarding the prospect of colistin resistance across MDR bacteria and its establishment via mutational or adaptation mechanisms. Besides, the swift emergence of bacterial resistance has further implicated the total reliance upon new antibiotics discovery, thus requiring strategic approaches towards implementing older antibiotics, colistin included.

\section{Acknowledgement}

The current work did not obtain any funding and was solely the authors' work. We would like to thank the staff of the Drug Applied Research Centre for their unwavering support.

\section{Author Contributions}

All authors contributed towards data analysis and the process of paper drafting and revision, as well as agreed to their accountability for all aspects of the work.

\section{Disclosure}

The authors report no conflicts of interest in this work. 


\section{References}

1. Pourhajibagher M, Hashemi FB, Pourakbari B, Aziemzadeh M, Bahador A (2016) Antimicrobial resistance of Acinetobacter baumannii to imipenem in Iran: A systematic review and meta-analysis. The Open Microbiology Journal 10: 32.

2. Tenover FC, McGowan JE (1996) Reasons for the emergence of antibiotic resistance. The American Journal of The Medical Sciences 311(1): 9-16.

3. Almasaudi SB (2018) Acinetobacter spp. as nosocomial pathogens: Epidemiology and resistance features. Saudi Journal of Biological Sciences 25(3): 586-596.

4. Maravić A, Skočibušić M, Fredotović Ž, Šamanić I, Cvjetan S, et al. (2016) Urban riverine environment is a source of multidrug-resistant and ESBL-producing clinically important Acinetobacter spp. Environmental Science and Pollution Research 23(4): 3525-3535.

5. Al Atrouni A, JolyGuillou ML, Hamze M, Kempf M (2016) Reservoirs of non-baumannii Acinetobacter species. Frontiers in Microbiology 7: 49.

6. Evans BA, Hamouda A, Amyes SG (2013) The rise of carbapenemresistant Acinetobacter baumannii. Current Pharmaceutical Design 19(2): 223-238.

7. Agodi A, Voulgari E, Barchitta M, Quattrocchi A, Bellocchi P, et al. (2014) Spread of a carbapenem-and colistin-resistant Acinetobacter baumannil ST2 clonal strain causing outbreaks in two Sicilian hospitals. Journal of Hospital Infection 86(4): 260-266.

8. Poirel L, Jayol A, Nordmann P (2017) Polymyxins: Antibacterial activity, susceptibility testing, and resistance mechanisms encoded by plasmids or chromosomes. Clinical Microbiology Reviews 30(2): 557-596.

9. Bialvaei AZ, Kouhsari E, SalehiAbargouei A, Amirmozafari N, Ramazanzadeh R, et al. (2017). Epidemiology of multidrug-resistant Acinetobacter baumannii strains in Iran: A systematic review and metaanalysis. Journal of Chemotherapy 29(6): 327-337.

10. (2019) World Health Organization. World Health Organization model list of essential medicines: 21st list 2019.

11. Velkov T, Thompson PE, Nation RL, Li J (2010) Structure activity relationships of polymyxin antibiotics. Journal of Medicinal Chemistry 53(5): 1898-1916.

12. Hancock RE, Scott MG (2000) The role of antimicrobial peptides in animal defenses. Proceedings of the National Academy of Sciences 97(16): 8856-8861.

13. Bojkovic J, Richie DL, Six DA, Rath CM, Sawyer WS, et al. (2016) Characterization of an Acinetobacter baumannii lptD deletion strain: permeability defects and response to inhibition of lipopolysaccharide and fatty acid biosynthesis. Journal of Bacteriology 198(4): 731-741.

14. Liu YY, Wang Y, Walsh TR, Yi LX, Zhang R, et al. (2016) Emergence of plasmid-mediated colistin resistance mechanism MCR-1 in animals and human beings in China: A microbiological and molecular biological study. The Lancet Infectious Diseases 16(2): 161-168.

15. Ahmed SS, Alp E, Hopman J, Voss A (2016) Global epidemiology on colistin resistant Acinetobacter baumannii. Journal of Infectious Diseases \& Therapy 35(9): 1469.

16. Da Silva GJ, Domingues S (2017) Interplay between colistin resistance, virulence and fitness in Acinetobacter baumannii. Antibiotics 6(4): 28.

17. Snitkin ES, Zelazny AM, Gupta J, Palmore TN, Murray PR (2013) Genomic insights into the fate of colistin resistance and Acinetobacter baumannii during patient treatment. Genome Research 23(7): 1155-1162.

18. Park YK, Choi JY, Shin D, Ko KS (2011) Correlation between overexpression and amino acid substitution of the PmrAB locus and colistin resistance in Acinetobacter baumannii. International Journal of Antimicrobial Agents 37(6): 525-530.

19. Pelletier MR, Casella LG, Jones JW, Adams MD, Zurawski DV, et al. (2013) Unique structural modifications are present in the lipopolysaccharide from colistin-resistant strains of Acinetobacter baumannii. Antimicrobial Agents and Chemotherapy 57(10): 4831-4840.

20. Boll JM, Tucker A., Klein DR, Beltran AM, Brodbelt JS (2015) Reinforcing lipid a acylation on the cell surface of Acinetobacter baumannii promotes cationic antimicrobial peptide resistance and desiccation survival. MBio 6(3): e00478-e00515.

21. Moffatt JH, Harper M, Adler B, Nation RL, Li J, et al. (2011) Insertion sequence ISAba11 is involved in colistin resistance and loss of lipopolysaccharide in Acinetobacter baumannii. Antimicrobial Agents and Chemotherapy 55(6): 3022-3024.

22. Lean SS, Suhaili Z, Ismail S, Rahman NIA, Othman N, et al. (2014) Prevalence and genetic characterization of carbapenem-and polymyxinresistant Acinetobacter baumannii isolated from a tertiary hospital in Terengganu, Malaysia. ISRN microbiology.

23. Bojkovic J, Richie DL, Six DA, Rath CM, Sawyer WS, et al. (2016) Characterization of an Acinetobacter baumannii lptD deletion strain: permeability defects and response to inhibition of lipopolysaccharide and fatty acid biosynthesis. Journal of Bacteriology 198(4): 731-741.

24. Moffatt JH, Harper M, Mansell A, Crane B, Fitzsimons TC, et al. (2013) Lipopolysaccharide-deficient Acinetobacter baumannii shows altered signaling through host Toll-like receptors and increased susceptibility to the host antimicrobial peptide LL-37. Infection and Immunity 81(3): 684-689.

25. Maifiah MhM, Cheah SE, Johnson MD, Han ML, Boyce JD, et al. (2016) Global metabolic analyses identify key differences in metabolite levels between polymyxin-susceptible and polymyxin-resistant Acinetobacter baumannii. Scientific Reports 6: 22287.

26. Malinverni JC, Silhavy TJ (2009) An ABC transport system that maintains lipid asymmetry in the gram-negative outer membrane. Proceedings of the National Academy of Sciences 106(19): 8009-8014.

27. Audet A, Nantel G, Proulx P (1974) Phospholipase a activity in growing Eacherichia coli cells. Biochimica et Biophysica Acta (BBA)-Lipids and Lipid Metabolism 348(3): 334-343.

28. Nhu NTK, Riordan DW, Nhu TDH, Thanh DP, Thwaites G, et al. (2016) The induction and identification of novel Colistin resistance mutations in Acinetobacter baumannii and their implications. Scientific Reports 6(1): 1-8.

29. Hood MI, Jacobs AC, Sayood K, Dunman PM, Skaar EP (2010) Acinetobacter baumannii increases tolerance to antibiotics in response to monovalent cations. Antimicrobial Agents and Chemotherapy 54(3): 1029-1041.

30. Ni W, Li Y, Guan J, Zhao J, Cui J, et al. (2016) Effects of efflux pump inhibitors on colistin resistance in multidrug-resistant Gram-negative bacteria. Antimicrobial Agents and Chemotherapy 60(5): 3215-3218.

31. Poirel L, Jayol A, Nordmann P (2017) Polymyxins: Antibacterial activity, susceptibility testing, and resistance mechanisms encoded by plasmids or chromosomes. Clinical Microbiology Reviews 30(2): 557-596.

32. Sherman EX, Hufnagel DA, Weiss DS (2016) MCR-1 confers crossresistance to lysozyme. The Lancet Infectious Diseases 16(11): 12261227.

33. Falgenhauer L, Waezsada SE, Yao Y, Imirzalioglu C, Käsbohrer A et al. (2016) Colistin resistance gene mcr-1 in extended-spectrum $\beta$-lactamase-producing and carbapenemase-producing Gram-negative bacteria in Germany. The Lancet Infectious Diseases 16(3): 282-283.

34. Perreten V, Strauss C, Collaud A, Gerber D (2016) Colistin resistance gene mcr-1 in avian pathogenic Escherichia coli in South Africa. Antimicrobial Agents and Chemotherapy 60(7): 4414-4415.

35. Poirel L, Nordmann P (2016) Emerging plasmid-encoded colistin resistance: The animal world as the culprit? Journal of Antimicrobial Chemotherapy 71(8): 2326-2327. 
36. Poirel L, Jayol A, Nordmann P (2017) Polymyxins: Antibacterial activity, susceptibility testing, and resistance mechanisms encoded by plasmids or chromosomes. Clinical Microbiology Reviews 30(2): 557-596.

37. Haenni M, Poirel L, Kieffer N, Châtre P, Saras E, et al. (2016) Cooccurrence of extended spectrum $\beta$ lactamase and MCR-1 encoding genes on plasmids. The Lancet Infectious Diseases 16(3): 281-282.

38. Du H, Chen L, Tang YW, Kreiswirth BN (2016) Emergence of the mcr1 colistin resistance gene in carbapenem-resistant Enterobacteriaceae. The Lancet Infectious Diseases 16(3): 287-288.

39. Yin W, Li H, Shen Y, Liu Z., Wang S, et al. (2017) Novel plasmid-mediated colistin resistance gene mcr-3 in Escherichia coli. MBio 8(3): 00543-17.

40. Borowiak M, Fischer J, Hammerl JA, Hendriksen RS, Szabo I, et al. (2017) Identification of a novel transposon-associated phosphoethanolamine transferase gene, mcr-5, conferring colistin resistance in d-tartrate fermenting Salmonella enterica subsp. enterica serovar Paratyphi B. Journal of Antimicrobial Chemotherapy 72(12): 3317-3324.

ISSN: 2574-1241

DOI: 10.26717/BJSTR.2020.29.004755

Zhang Jieling, Li Honglang. Biomed J Sci \& Tech Res

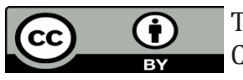

This work is licensed under Creative Commons Attribution 4.0 License

Submission Link: https://biomedres.us/submit-manuscript.php
41. Abuoun M, Stubberfield EJ, Duggett NA, Kirchner M, Dormer L, et al. (2017) mcr-1 and mcr-2 variant genes identified in Moraxella species isolated from pigs in Great Britain from 2014 to 2015. Journal of Antimicrobial Chemotherapy 72(10): 2745-2749.

42. Yang YQ Li YX, Lei CW, Zhang AY, Wang HN (2018) Novel plasmidmediated colistin resistance gene mcr-7.1 in Klebsiella pneumoniae. Journal of Antimicrobial Chemotherapy 73(7): 1791-1795.

43. Wang X, Wang Y, Zhou Y, Li J, Yin W, et al. (2018) Emergence of a novel mobile colistin resistance gene, mcr-8, in NDM-producing Klebsiella pneumoniae. Emerging Microbes \& Infections 7(1): 1-9.

44. Kieffer N, Nordmann P, Poirel L (2017) Moraxella species as potential sources of MCR-like polymyxin resistance determinants. Antimicrobial Agents and Chemotherapy 61(6): e00129-17.

45.Zurfluh K, Tasara T, Poirel L, Nordmann P, Stephan R (2016) Draft genome sequence of Escherichia coli S51, a chicken isolate harboring a chromosomally encoded mcr-1 gene. Genome Announc 4(4): e0079616.

$\begin{array}{ll}\text { BIOMEDICAL } & \text { Assets of Publishing with us } \\ \text { RESEARCHES } & \text { - Global archiving of articles } \\ & \text { - Immediate, unrestricted online access } \\ \end{array}$

